\title{
On testing neural network models
}

\section{Rafael Yuste}

In my recent Timeline article, I described the emergence of neural network models as an important paradigm in neuroscience research (From the neuron doctrine to neural networks. Nat. Rev. Neurosci. 16, 487-497 $(2015))^{1}$. In his correspondence (Neural networks in the future of neuroscience research. Nat. Rev. Neurosci. http://dx.doi. org/10.1038/nrn4042 (2015)) $)^{2}$, Rubinov provides some thoughtful comments about the distinction between artificial neural networks and biologically inspired ones and about how a strictly data-driven approach may succeed at providing a general theory of neural circuits. I thank Rubinov for these comments and note that this theory agnosticism is a methodological approach that we respect and indeed sponsored in our Brain Activity Map proposal that led to the BRAIN Initiative $e^{3}$. Also, although in my Timeline article I tried to provide a brief summary of the history of artificial neural network models, I am not yet personally convinced that there are clear instances in which a biologically inspired neural network model has yet been validated (“...it is unclear whether existing neural network models have enough predictive value to be considered valid or useful for explaining brain circuits." (REF. 1)). There are many exciting areas of progress in current neuroscience detailing phenomenology that is consistent with some neural network models, some of which I tried to summarize and illustrate, but at the same time we are still far from a rigorous demonstration of any neural network model with causal experiments. I therefore could not agree more with Rubinov that we still have "largely not bridged the gap between elegant theory and neuroscientific observation". But when will we know that we have bridged that gap? This is a difficult question to answer, depending on the particular viewpoint, and I would leave this open to the reader's own interpretation. In my mind, a successful neural model should have quantitative accuracy in predicting either the behaviour, mental or perceptual state of the animal, or at least the future internal dynamics of the system. Another characteristic of a successful model could be its effective use in designing therapies of brain-based diseases. On the other hand, one of my mentors, David Tank, argued that for a true understanding of a neural circuit we should be able to actually build it, which is a stricter definition of a successful theory (D. Tank, personal communication) Finally, as mentioned in the Timeline article, one will also need to connect neural network models to theories and facts at the structural and biophysical levels of neural circuits and to those in cognitive sciences as well, for proper 'scientific knowledge' to occur in the Kantian sense.
Rafael Yuste is at the Neurotechnology Center and Kavli Institute of Brain Sciences, Departments of Biological Sciences and Neuroscience, Columbia University, New York, New York 10027, USA.
e-mail: rmy5@columbia.edu
doi: $10.1038 / \mathrm{nrn} 4043$ Published online 21 October 2015

1. Yuste, R. From the neuron doctrine to neural networks. Nat. Rev. Neurosci. 16, 487-497 (2015).

2. Rubinov, M. Neural networks in the future of neuroscience research. Nat. Rev. Neurosci. http://dx. doi.org/10.1038/nrn4042 (2015).

3. Alivisatos, A. P. et al. The brain activity map project and the challenge of functional connectomics. Neuron 74, 970-974 (2012).

\section{Acknowledgements}

The author is supported by the US National Institutes of Health (DP1EY024503) and ARO W911NF-12-1-0594 (MURI).

Competing interests statement

The author declares no competing interests. 